\title{
Specific Immune Response Induced by a Lactobacillus Associated with a Pneumococcal Antigen in an "in vitro" Human Cells Model
}

\section{Elisa Vintiñi ${ }^{1,2}$, Laura Gonzalez ${ }^{3}$ and Marcela Medina ${ }^{2,4 *}$}

${ }^{1}$ Facultad de Agronomía y Zootecnia, Florentino Ameghino S/N, El Manantial, 4105, Tucumán, Argentina ${ }^{2}$ Centro de Referencia para Lactobacilos (CERELA-CONICET). Chacabuco 145. 4000. Tucumán. Argentina

${ }^{3}$ Facultad de Medicina, Av, Roca 1900.4000, Universidad Nacional de Tucumán, Tucumán, Argentina

${ }^{4}$ Facultad de Bioquímica, Química y Farmacia. Ayacucho 471. 4000. Universidad Nacional de Tucumán. San Miguel de Tucumán. Tucumán. Argentina.

\begin{abstract}
In this work we assessed the immune response induced by combinations of live (LCV) and heat-killed (LCM) Lactobacillus casei CRL 431 as adjuvants associated with pneumococcal protective A protein (PppA) in peripheral blood mononuclear cells (PBMCs). LcV, LcM and their combinations with PppA stimulated T, B and NK cells. Thus, all stimuli increased CD25 expression in T CD3 lymphocytes, highest activation being reached with the combinations of $\mathrm{LcV}$ or LcM with an antigen (PppA+LcV, PppA+LcM). Expression of CD19 B cells marker was significantly increased in almost all treatments compared with non-stimulated PBMCs, except for PppA. All treatments increased CD86 expression in the LT population, while in B cells only LPS, PppA+LcV and PppA+LcM increased it. NK cells were significantly increased by LPS $(P<0.05), P p p A+L c M(P<0.01)$ and $P p p A+L c V(P<0.01)$ compared to non-stimulated PBMCs. PppA+LcV and PppA+LcM increased CD56 expression in both NKT and NK cells, while LcM expanded NKT population. Cytokine pattern analysis showed that LcV and LcM stimulated Th, Th2 and Th17 cytokines and exerted an important adjuvant effect when associated with PppA. Correlation with previous results obtained in animal models when the same experimental vaccine was nasally administered is discussed. Human PBMCs would be useful to evaluate the immune response of mucosal vaccines containing lactic acid bacteria associated with a specific antigen.
\end{abstract}

Keywords: Heat-killed lactobacilli; Pneumococcal antigen; Vaccine, PBMCs; Immune response

Abbreviations: Lc: Lactobacillus casei; LcV: Live Lactobacillus casei; LcM: Heat-killed Lactobacillus casei; PBMCs: Peripheral blood mononuclear cells; NK: Natural Killer; NKT: Natural Killer T; PppA: Pneumococcal protective A protein; LT: T cells; LB: B cells; IFN- $\gamma$ : Interferon Gamma; IL-4: Interleukin 4; IL-2: Interleukin 2; IL-10: Interleukin 10; IL-17 Interleukin 17; Th: T elper

\section{Introduction}

Vaccines are the main option to prevent most infectious diseases, pneumococcal infections being one of the most important public health issues in the world $[1,2]$. The existing pneumococcal vaccines, capsular polysaccharide pneumococcal vaccines (PPV) and proteinpolysaccharide conjugate pneumococcal vaccines (PCV), have failed to prevent pneumococcal infections because the former are not effective in at-risk populations and the latter are too expensive to be used in developing countries. Pneumococcal proteins conserved among various serotypes have been evaluated and represent a new alternative for the development of protein-based pneumococcal vaccines (PbPVs) $[3,4]$, but at present no PbPVs have been licensed. In addition, while S. pneumoniae enters the body by the nasal route, all vaccines are administered by the parenteral pathway and induce a good specifi humoral response at the systemic level but not in mucosa. A crucial topic in the development of a pneumococcal vaccine is the selection of appropriate adjuvants that can enhance both the humoral and the cellular immune response. The most important consideration in the selection of an appropriate adjuvant for use in human vaccines is its biosafety and the only adjuvants approved for human use are aluminum salts. These adjuvants, which are used in parenteral vaccines to increase the titer of specific antibodies, are not the optimal adjuvants to induce CD4+ Th1 cells or cytotoxic (T or NK cells) immune responses, which are very important in the control of various pathogens. For mucosal vaccines, the most effective experimental mucosal adjuvants known today are the cholera toxin and E. coli lymphotoxin, which are able to induce a good systemic and mucosal immune response. However, they are also very toxic and not acceptable for human use [5] despite attempts to overcome this drawback [6,7]. Within this context, the use of probiotic lactic acid bacteria (LAB) as mucosal adjuvants emerges as a novel alternative to enhance the specific immune response. There is vast evidence showing that the main mechanism through which probiotics provide health benefits is the modulation of immune functions, an effect that is strain-dependent [8,9]. Lactobacillus casei CRL 431 is a probiotic as well as an immunobiotic [10] strain since it is able to activate the mucosal immune system $[8,9,11]$. Previous reports have shown that this lactobacillus was able to increase specific anti-pneumococcal IgA and IgG after a respiratory pneumococcal infection in a mouse model. In addition, pro-inflammatory TNF- $\alpha$ and regulatory IL-10 cytokines [11] increased when $L$. case $i$ was preventively administered by the oral and nasal route $[11,12]$. In the development of vaccines, $\mathrm{LAB}$ were used mainly as antigen vehicles $[13,14]$ while there are few studies concerning the use of non-recombinant strains as adjuvants in mucosal vaccine formulations. In this sense, it was demonstrated that nasal administration of live and heat-killed $L$. case

*Corresponding author: Marcela Medina, CERELA, Chacabuco 145, Facultad de Bioquímica, Química y Farmacia, Facultad de Bioquímica, Química y Farmacia, Ayacucho 471.400, Universidad Nacional de Tucumán, San Miguel de Tucumán, Tucumán, Argentina, Tel: 54-0381-155753848; E-mail: marcemedina74@yahoo.com.ar

Received September 11, 2012; Accepted October 12, 2012; Published October 16,2012

Citation: Vintiñi E, Gonzalez L, Medina M (2012) Specific Immune Response Induced by a Lactobacillus Associated with a Pneumococcal Antigen in an "in vitro" Human Cells Model. J Vaccines Vaccin 3:153. doi:10.4172/2157-7560.1000153

Copyright: (C) 2012 Vintiñi E, et al. This is an open-access article distributed under the terms of the Creative Commons Attribution License, which permits unrestricted use, distribution, and reproduction in any medium, provided the original author and source are credited. 
CRL 431 associated with pneumococcal protective A protein (PppA) effectively prevented lung colonization in a pneumococcal respiratory infection and increased the survival of mice intraperitoneally challenged with the pathogens [15]. Animal models are very useful tools to study the effect of vaccines on the protection against human pathogens but the results obtained with them do not guarantee their effectiveness in human health. A recent report showed that previous diet supplementation with BB-12 or with $L$. casei 431 administered by the oral route effectively improved the immune function by increasing the systemic and mucosal immune response in volunteers before they received seasonal influenza vaccination [16]. No data are available with respect to the effect of immunobiotics strains associated with a specific antigen on cellular immunity, either from human studies or from "in vitro" assays in human cells. The aim of this work was to evaluate the immune response induced by experimental vaccines containing live or heat-killed lactobacilli as adjuvants associated with a pneumococcal antigen on cytokine production and cellular immunity in a human cells model (peripheral blood mononuclear cells: PBMCs) and to analyze the potential mechanism/s involved in this effect. On the other hand, in a previous work [15], the same vaccines administered nasally were evaluated in an animal model. Thus, correlation of results obtained "in vitro" and "in vivo" will be discussed in order to analyze the usefulness of PBMCs as an alternative to assess the effect of a mucosal vaccine on humans. Although PBMCs are a mixture of systemic immune cells, the immune response induced in them is more representative of the real situation of the body than the study of specific isolated mucosal cells. Moreover, mucosal vaccines are also required to provide systemic protection.

\section{Materials and Methods}

\section{Microorganisms and culture conditions}

Recombinant E. coli-PppA was obtained in our laboratory and the development of this strain was described in a previous report from our work group [17]. From this strain rPppA protein was purifi d. The development of this strain, from which recombinant PppA (rPppA) was purifi $\mathrm{d}$, was described in a previous report from our work group [17].

Lactobacillus casei CRL 431 (L. casei) [15,18], obtained from the CERELA culture collection, was cultured overnight at $37^{\circ} \mathrm{C}$ (fi al log phase) in Man-Rogosa-Sharpe broth (Oxoid), harvested and washed twice with sterile $0.01 \mathrm{M}$ phosphate buffer saline (PBS), $\mathrm{pH} 7.2$, and resuspended in PBS containing 20\% glycerol. Aliquots of these suspensions were stored at $-80^{\circ} \mathrm{C}$ until use. The number of live cells after freezing and thawing was determined by colony-forming unit (CFU) counting on MRS-C agar after $48 \mathrm{~h}$ incubation. 92-94\% cells of L. casei were alive upon thawing and no signifi ant differences were found during storage time ( 4 months). One fresh aliquot was thawed for every new experiment to prevent variability in the cultures between experiments. The bacterial suspension was adjusted to the desired concentration (106 cell/well) to stimulate PBMCs and this suspension constituted the live-cell suspensions: LcV. Heat-killed L. casei (LcM) was prepared by heating bacteria in a water bath at $80^{\circ} \mathrm{C}$ for $30 \mathrm{~min}$ and lack of bacterial growth was confi med using MRS agar plates.

\section{Isolation and stimulation of PBMCs}

PBMCs were isolated from the heparinized peripheral blood of four healthy volunteers (median age 28 years, range $25-33$ years) after their written consent was obtained, as described in a previous report [19]. Briefly, PBMCs were isolated by centrifugation over a Ficoll density gradient (SIGMA, NJ, USA), washed with RPMI-1640 (Gibco, Invitrogen, Buenos Aires, Argentina) and adjusted to $1 \times 106$ cells $/ \mathrm{ml}$ in RPMI-1640 supplemented with 10\% fetal bovine serum (FBS) (Natacor, Córdoba, Argentina), $2 \mathrm{mM}$ l-glutamine, $100 \mathrm{mg} / \mathrm{ml}$ streptomycin and $100 \mathrm{U} / \mathrm{ml}$ penicillin (Sigma). PBMCs were incubated in 24-well flatbottomed polystyrene microtiter plates (Corning, GBO, Argentina) in the presence or absence of different stimulants in a humidifi $\mathrm{d}$ atmosphere of $5 \% \mathrm{CO}_{2}$ at $37^{\circ} \mathrm{C}$ for $24 \mathrm{~h}$. Final concentrations of either $1 \times 106 \mathrm{CFU} / \mathrm{ml}$ live and heat-killed cell suspensions, PppA $(10 \mu \mathrm{g} / \mathrm{ml})$ or combinations of LcV or LcM plus PppA were used as stimulants. Purifi d lipopolysaccharide (LPS) from Escherichia coli O111:B4 (Sigma) was used to stimulate PBMCs at a concentration of $1 \mu \mathrm{g} / \mathrm{ml}$ as a positive control. Non-stimulated PBMCs were also evaluated as controls of basal cytokine production and cell-surface marker expression. All reagents were tested by the E-toxate test for LPS (Sigma) and shown to be below the detection limit $(2 \mathrm{pg} / \mathrm{ml})$.

Every stimulus was assayed in duplicate and each experiment was performed with PBMCs from four donors. Cell culture supernatants were collected by centrifugation, filtered using $0.22 \mu \mathrm{l}$ filters that eliminate bacteria, fractionated in aliquots and stored at $-20^{\circ} \mathrm{C}$ until cytokines were analyzed. PBMCs were harvested for cytometry analysis as described below.

\section{Pneumococcal antigen}

rPppA was purifi d from E. coli-PppA [17] using a His-Bind purifi ation kit (Novagen) and visualized by electrophoresis on $12 \%$ SDS-polyacrylamide gels, as previously described [17]. The reagent and the PppA solution were tested by the E-toxate test for LPS (Sigma) and shown to be below the limit of detection $(2 \mathrm{pg} / \mathrm{ml})$. rPppA concentration was determined by Bradford's method and 10 ug of this protein was used in immunization protocols. Th s same concentration of PppA was employed in a previous report in an animal model [15].

\section{PBMC surface phenotyping by flow cytometry}

To evaluate the effects of live or heat-killed L. casei, PppA and combinations of both on PBMC surface antigen expression, cells were removed gently from wells by scraping and stained with appropriate combinations of fluorescently labeled monoclonal antibodies (mAbs) that included: fluorescein isothiocyanate (FITC)-labeled anti-CD3, anti-CD19, anti-CD86; phycoerythrin (PE)-labeled anti-CD4, antiCD25 and peridinin chlorophyll-aprotein (PercP) anti-CD8. In addition, triple CD4-CD8 and CD3 mAbs were used when T cells were evaluated (all $\mathrm{mAbs}$ from BD Pharmigen). Cells were stained with labeled $\mathrm{mAbs}$ in PBS $+2 \%$ fetal bovine serum (PBS-FBS) for $30 \mathrm{~min}$ at $4^{\circ} \mathrm{C}$, washed and re-suspended in ice-cold PBS-FBS. Then, cells were acquired by a Partec Past II fl w cytometer (BD Bioscience) and data were analyzed with Flomax software.

\section{Cytokine assays}

Cytokine concentrations of supernatants were measured by human Th1/Th enzyme-linked immunosorbent assay (ELISA) Ready SET Go! Kit (BD Bioscience, San Diego, CA, USA), including interleukin (IL)2 and interferon (IFN) $-\gamma$ as Th1- ype and IL- 4 and IL-10 as Th2- ype cytokines. The IL-17 cytokine was also measured using the ELISA kit from e-Bioscience (BD Biosciences). The detection procedures were performed according to the manufacturer's instructions. The sensitivity of the assays for each cytokine was as follows: $4 \mathrm{pg} / \mathrm{ml}$ for IL- 2 , IFN- $\gamma$ and IL-17, and $2 \mathrm{pg} / \mathrm{ml}$ for IL-4 and IL-10. 
Citation: Vintiñi E, Gonzalez L, Medina M (2012) Specific Immune Response Induced by a Lactobacillus Associated with a Pneumococcal Antigen in an "in vitro" Human Cells Model. J Vaccines Vaccin 3:153. doi:10.4172/2157-7560.1000153

\section{Statistical analyses}

Experiments were performed in triplicate and results were expressed as mean \pm standard deviation (SD). Data were evaluated by one-way or two-way ANOVA tests. Tukey's test (for pairwise

\begin{tabular}{|l|c|c|c|c|c|}
\hline & CD3+ & CD4+ & CD8+ & CD3+CD86+ & CD25+CD3+ \\
\hline Control & $70.6 \pm 205$ & $44.1 \pm 3.5$ & $25.3 \pm 1.2$ & $0.76 \pm 0.12^{\mathrm{a}}$ & $1.60 \pm 0.21^{\mathrm{a}}$ \\
\hline LPS & $71.7 \pm 1.8$ & $42.7 \pm 5.7$ & $25.3 \pm 1.3$ & $1.38 \pm 0.15^{\mathrm{b}}$ & $2.90 \pm 0.90^{\mathrm{b}}$ \\
\hline LcV & $71.2 \pm 2.4$ & $45.2 \pm 3.2$ & $25.1 \pm 1.1$ & $0.96 \pm 0.10^{\mathrm{c}}$ & $3.10 \pm 0.8^{\mathrm{b}}$ \\
\hline LcM & $69.5 \pm 1.6$ & $45.4 \pm 4.2$ & $24.1 \pm 1.5$ & $0.94 \pm 0.07^{\mathrm{c}}$ & $3.34 \pm 0.67^{\mathrm{b}}$ \\
\hline PppA & $69.4 \pm 3.5$ & $45.1 \pm 4.7$ & $25.7 \pm 1.3$ & $0.89 \pm 0.05^{\mathrm{c}}$ & $2.69 \pm 0.53^{\mathrm{b}}$ \\
\hline PppA+LcV & $71.0 \pm 1.8$ & $47.1 \pm 2.1$ & $26.2 \pm 1.6$ & $1.02 \pm 0.02^{\mathrm{d}}$ & $3.90 \pm 0.84^{\mathrm{c}}$ \\
\hline PppA_LcM & $70.9 \pm 2.2$ & $46.4 \pm 3.8$ & $23.1 \pm 1.8$ & $0.99 \pm 0.01^{\mathrm{d}}$ & $3.67 \pm 0.81^{\mathrm{c}}$ \\
\hline
\end{tabular}

Table 1: Surface cell markers in T human cells.

\begin{tabular}{|l|c|c|}
\hline & CD19+ & CD19+CD86+ \\
\hline Control & $10 \pm 1,5^{\mathrm{a}}$ & $0.44 \pm 0.01^{\mathrm{a}}$ \\
\hline LPS & $13 \pm 1,1^{\mathrm{b}}$ & $0.75 \pm 0.10^{\mathrm{b}}$ \\
\hline LcV & $12,5 \pm 1,3^{\mathrm{b}}$ & $0.49 \pm 0.04^{\mathrm{c}}$ \\
\hline LcM & $13,5 \pm 1,4^{\mathrm{b}}$ & $0.48 \pm 0.02^{\mathrm{c}}$ \\
\hline PppA & $11,8 \pm 1,0^{\mathrm{a}}$ & $0.42 \pm 0.03^{\mathrm{a}}$ \\
\hline PppA+LcV & $13,4 \pm 1,3^{\mathrm{b}}$ & $0.52 \pm 0.03^{\mathrm{d}}$ \\
\hline PppA+LcM & $13,9 \pm 1,5^{\mathrm{b}}$ & $0.52 \pm 0.02^{\mathrm{d}}$ \\
\hline
\end{tabular}

Expression of surface markers CD19 and CD86 induced by peripheral blood mononuclear cells stimulated with live (LcV), heat-killed Lactobacillus casei (LCM), pneumococcal antigen (PppA), combinations of both Lc and PppA (PppA+LcV, PppA+LcM), LPS and Control. (See Table 1 for details)

Table 2: Surface cell markers of B cells. comparisons of the mean values of the different groups) was used to test for differences between the groups. Significant difference was defined as $P<0.05$

\section{Results}

T- and B-cell surface marker expression and activation induced by experimental vaccine

Both humoral and cellular immune responses are important components of the defense immune system in the struggle against S. pneumoniae. Thus, we studied the effect of live $(\mathrm{LcV})$ or heatkilled (LcM) L. casei in combination with a pneumococcal antigen on the expression of $\mathrm{T}$ and $\mathrm{B}$ surface markers. With respect to the $\mathrm{T}$ lymphocyte population, results showed that none of the stimuli assayed induced changes in the expression of cell surface marker CD3; thus, total $\mathrm{T}$ population in all treatments showed expression values similar to those of the control (RPMI) (Table 1). In addition, we determined whether the expression of $\mathrm{CD} 4$ (TCD4+ $=$ helper $\mathrm{T}$ lymphocytes) or CD8 (TCD8+ = cytotoxic T lymphocytes) was modifi d. Results demonstrated that none of the stimuli assayed was able to increase CD4 or CD8 expression in PBMCs. In order to evaluate the effect of different stimuli on total B lymphocytes, we used CD19 as a marker of mature B cells. In contrast to T cells, CD19 expression was signifi antly increased in almost all the treatments compared with control, the only exception being the PppA group (Table 2). Activation of B and T cells after specific stimulation was evaluated by the expression of the CD86 molecule in these cells. The CD86 molecule is expressed on activated $\mathrm{B}$ cells, T cells, dendritic cells and monocytes/macrophages. Analysis of CD86+CD3+ T cells and CD86+CD19 B cells (Table 1 and Table
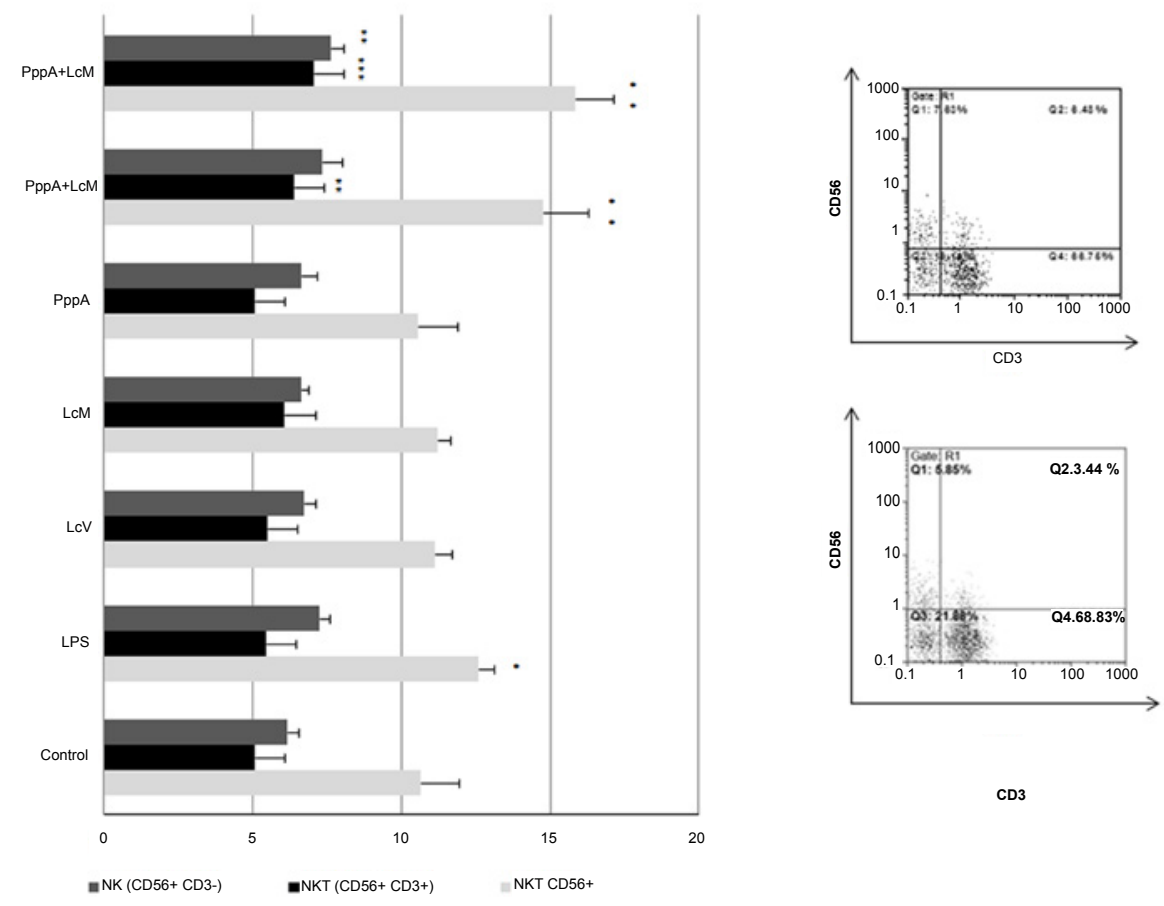

$\mathrm{CD} 3$

Figure 1: Effect of live (LcV), heat-killed Lactobacillus casei (LcM), pneumococcal antigen (PppA), combinations of both Lc and PppA (PppA+LcV, $P p p A+L c M)$ and LPS on expression of surface markers CD56 and CD3 in peripheral blood mononuclear cells. $A) C o n t r o l$ and $B) P p p A+L c M$ are representatives dot-plot using anti-CD3 and anti-CD56 mAb, allowing identification of 3 cell subsets: CD3-CD56+ (NK cells), CD3+CD56+ (NK-T cells) and CD3+CD56- (T cells). Graphic indicates the percentage of cells for each of these subsets. Data are expressed as mean \pm ds values of 4 healthy donors. Duplicate assays were performed for each donor and each stimulus. $\left({ }^{\star} P<0.05,{ }^{* *} P<0.01,{ }^{* * *} P<0.001\right)$. 
2) showed that all treatments increased CD86 expression in the LT population, while in the LB population only LPS, PppA+LcV and $\mathrm{PppA}+\mathrm{LcM}$ induced an increased expression of that marker. Overall, these results indicate that the experimental vaccines were able to induce the activation of $\mathrm{T}$ and $\mathrm{B}$ cells with expansion of $\mathrm{B}$ cells and that $\mathrm{LcV}$ or LcM exerted an adjuvant effect when combined with the pneumococcal antigen. Humoral immunity is an important component of the defense against pneumococci and B activation requires the cooperation of LT. Thus, in order to evaluate the antigen-primed specific $\mathrm{T}$ cells after stimulation, we studied the activation of $\mathrm{T}$ CD3 + cells by evaluating CD25 marker expression (Table 1). 1.6\% of the non-stimulated CD3+ T cells expressed the surface CD25 marker while all the stimuli were able to induce an increase in CD25 expression on T lymphocytes. However, highest activation of $\mathrm{CD} 3+\mathrm{T}$ cells was reached by the combinations of $\mathrm{LcV}$ and LcM with the pneumococcal antigen. These groups reached an increase in CD25 expression of above 100\% compared with control PBMCs.

\section{Evaluation of surface markers of NK cells}

NK cells are considered as a part of the innate immunity but they also represent a nexus between innate and adaptive immunity through the secretion of cytokines such as INF- $\gamma$ and TNF- $\alpha$. The CD56 marker was used to characterize this NK population by fl $\mathrm{w}$ cytometry (Figure 1). Results showed that surface marker CD56 expression that included total NK cells was signifi antly increased only by PBMC stimulation with LPS $(P<0.05)$, PppA+LcM $(P<0.01)$ and PppA+LcV $(P<0.01)$ compared to control, while $\mathrm{LcV}$ and LcM tended to increase surface marker CD56 expression but no signifi ant differences were found. In order to discriminate between NK and NKT cells, we evaluated CD56+CD3- (NK) and CD56+CD3+ (NKT) cells. Stimulation with the combinations $\mathrm{PppA}+\mathrm{LcV}$ and $\mathrm{PppA}+\mathrm{LcM}$ increased CD56 expression
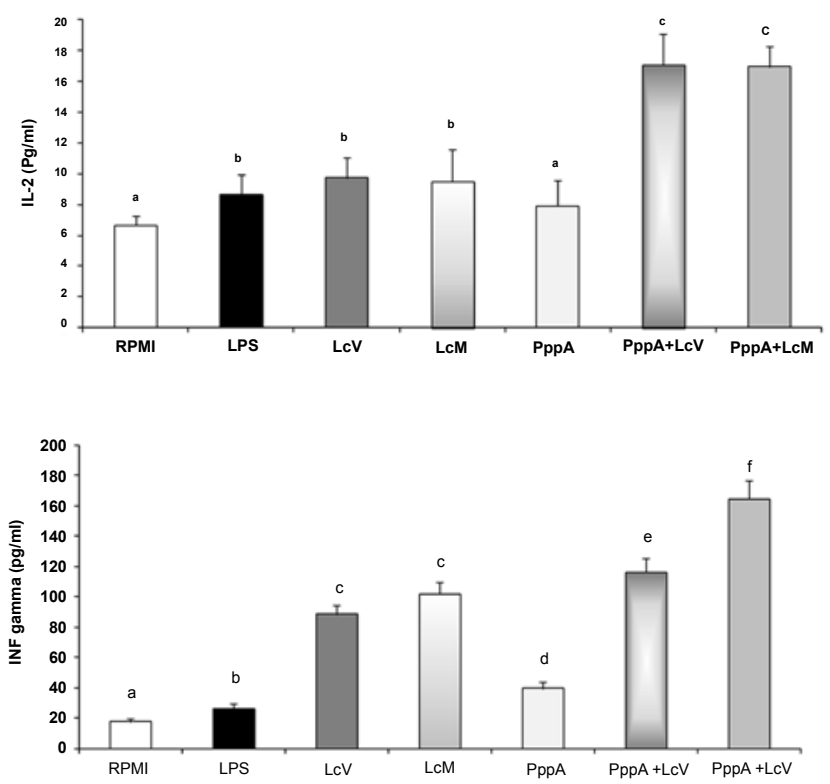

Figure 2: Th1 cytokines production by peripheral blood mononuclear cells stimulated with live (LcV), heat-killed Lactobacillus casei (LcM), pneumococcal antigen (PppA), combinations of both (PppA+LcV, PppA+LcM), LPS and Control. Panel A, IL-2 production, Panel B, INF-Y production. Results are expressed as mean \pm SD of duplicate measurements determined in four independent experiments. Tukey's test was used to test for differences between the groups. Means in the figure with different letters (a-f) were significantly different $(P<0.01)$.

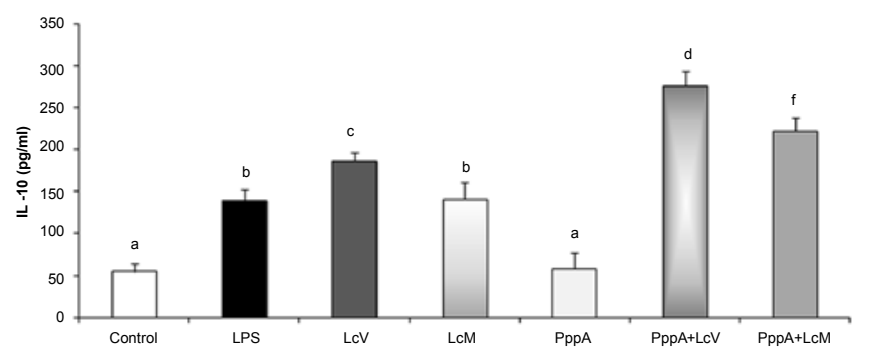

Figure 3: IL-10 production by peripheral blood mononuclear cells stimulated with live (LcV), heat-killed Lactobacillus casei (LcM), pneumococcal antigen (PppA), combinations of both (PppA+LcV, PppA+LcM), LPS and Control. (See Figure 2 for details).

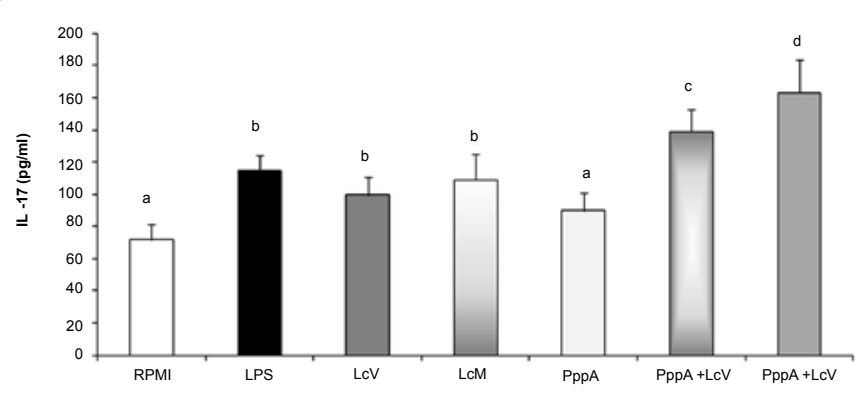

Figure 4: IL-17 production by peripheral blood mononuclear cells stimulated with live (LcV), heat-killed Lactobacillus casei (LcM), pneumococcal antigen (PppA), combinations of both (PppA+LcV, PppA+LcM), LPS and Control. (See Figure 2 for details).

in both NKT (CD3+CD56+) and NK (CD3-CD56+) cells, while LcM expanded NKT population in contrast with LPS, which expanded CD56+CD3- NK cells. LcV tended to expand this last population but no signifi ant differences were found compared with the control group.

Cytokine patterns induced by stimulation with experimental vaccines

In order to evaluate the activation of $\mathrm{T}$ cells, the patterns of cytokine production of Th1 (IL-2, INF- $\gamma$ ), Th2 (IL-4, IL-10) and Th17 (IL-17) induced by both LcV and LcM, pneumococcal antigen (PppA), probiotic plus PppA, LPS and RPMI (control) by PBMCs stimulation are shown in Figures 1, 2 and 3. Pro-inflammatory INF- $\gamma$ production was increased by all stimuli but PppA+LcV and PppA+LcM induced signifi antly higher values than the other stimuli (Figure 2). In addition, PppA+LcM induced signifi antly higher INF- $\gamma$ levels than PppA $+\mathrm{LcV}$ $(P<0.01)$. The T helper type 1 (Th1) IL-2 cytokine was signifi antly increased when cells were stimulated with almost all stimuli compared with control, the only exception being the PppA group. T helper type 2 (Th2) IL-4 and IL-10 cytokine production was also evaluated. Stimulation of PBMCs "in vitro" resulted in the production of very low amounts of IL-4. Thus, control cells (RPMI) and PppA groups showed no production of this cytokine. In contrast, LPS, LcV, LcM, LcV+PppA and $\mathrm{LcM}+\mathrm{PppA}$ were able to induce a signifi ant increase in IL-4 levels: $\mathrm{pg} / \mathrm{ml}: \mathrm{LPS}=6.7^{\star} \pm 1.2(P<0.01) ; \mathrm{LcV}=9.7^{\star} \pm 1.3(P<0.01) ; \mathrm{LcM}=9.5^{\star} \pm 1.6$ $(P<0.001) ; \quad \mathrm{LcV}+\mathrm{PppA}=17.1^{* *} \pm 2 \quad(P<0.001) ; \quad \mathrm{LcM}+\mathrm{PppA}=16.9^{* *} \pm 3$ $(P<0.01)$. The induction of regulatory IL-10 cytokine was increased by all the stimuli except PppA (Figure 3). An interesting fact was that LcV induced higher IL-10 production than $\operatorname{LcM}(P<0.01)$ and this behavior was maintained when combinations with PppA were evaluated. The IL-17A cytokine pattern was also evaluated and results showed that all 
the stimuli tested induced increased levels of this cytokine compared with control (Figure 4). Overall, LcV, LcM and their combinations with PppA increased Th1, Th2 and Th17 cytokines while PppA only induced an increase in INF- $\gamma$ and IL-17 levels. Live and heat-killed L. casei were able to stimulate Th1, Th2 and Th17 cytokines and exerted an important adjuvant effect when associated with the pneumococcal antigen.

\section{Discussion}

The development of effective broad-coverage pneumococcal vaccines is an important challenge nowadays. The use of pneumococcal proteins, independently of serotypes, is the most promising alternative, although so far there are no licensed vaccines based on these antigens. Some relevant aspects to be considered in this respect are the route of immunization, the efficacy and immunogenicity of the vaccine and the adjuvants employed. Most vaccines are delivered through the parenteral route. However, mucosal vaccination has several benefits over the parenteral way: it is easy to administer, it can be administered through the same route of entry as pathogens, it makes self-administration possible and is able to induce the systemic and mucosal immune response. The nasal route is undoubtedly the better way to provide immunization against pathogens that enter the body through the respiratory tract and generally an appropriate immune response in the mucosal and systemic compartments is induced against specific antigens $[5,20,21]$. However, in contrast to parenteral immunization, mucosal immunizations require potent adjuvants to enhance immunogenicity. At present, numerous investigations aimed at selecting appropriate mucosal adjuvants are being conducted around the world [21,22]. However, only a few adjuvants have been accepted for use in human health and some of them have undesirable side effects $[5,21]$. Thus, safety is a crucial characteristic in the selection of potential mucosal adjuvants. Lactic acid bacteria are considered GRAS (Generally Recognized as Safe) microorganisms and their capacity to stimulate the immune system is amply documented, so that they are good candidates to be used as mucosal adjuvants. In previous reports, the immunostimulant properties of an inactivated recombinant lactococcus expressing PppA [18] and live/heat-killed Lactobacillus casei associated with PppA were demonstrated in mice [15]. The animal model allowed us to obtain crucial information about the mucosal adjuvant effect of dead and live Lactobacillus casei. However, the results obtained did not guarantee the same positive effect on the human immune system. In this work, the effect of the combination of a pneumococcal antigen with immunobiotic lactobacilli on human immunity was evaluated using primary cultures of human PBMCs. Different immune cells were evaluated after stimulation with experimental vaccine formulations. $T$ cells play a crucial role in the mechanisms involved in the humoral and cellular specific immune response induced after vaccination. In the PBMCs model, combinations of $\mathrm{LcV}$ and $\mathrm{LcM}$ with PppA were able to induce an increase in the expression of the surface marker CD25. CD25 (IL-2Ra) is a marker expressed principally on activated T cells and Th1, Th2 and Th17 cytokines production induced by almost all stimuli was also a clear evidence of $\mathrm{T}$ cells activation. On the other hand, previous reports have shown overall agreement with $\mathrm{CD} 25$ and proliferation assays when a recall specific antigen was used $[23,24]$. In our model, CD3, CD4 and CD8 markers did not increase their expression when PBMCs were stimulated. It seems likely that a longer stimulation period is necessary to induce a signifi ant clonal expansion of $\mathrm{T}$ cells compared with control without stimulation [25].

With respect to CD19+ B cells, these were increased with almost all stimuli except PppA. Highest CD19 expression was obtained by stimulation with PppA+LcV and PppA+LcM, while $\mathrm{LcV}$ and $\mathrm{LcM}$ were also able to increase the expression of this marker, with no signifi ant differences between them. In addition, surface marker CD86 expression was increased by all stimuli in T cells while in B cells the stimulation with PppA was not able to increase CD86 expression. T-cell-B-cell cooperation is a crucial event in the induction of the specific immune response against protein antigens and both $\mathrm{LcV}$ and $\mathrm{LcM}$ exerted a stimulant effect on the activation of both $\mathrm{T}$ and $\mathrm{B}$ cells. The humoral immune response plays a fundamental role in vaccination for the generation of specific antibodies. In a previous report, $\mathrm{PppA}+\mathrm{LcV}$ and PppA+LcM combinations, nasally administered in a mouse model, were able to induce high IgG and IgA anti-PppA levels in systemic and mucosal compartments and these antibodies remained elevated up to and including day 74 [15]. In the "in vitro" model used in this work, IL-4 production showed higher levels than the control groups in all cases, except for PppA. IL-4 is a pleiotropic cytokine that plays a key role in the activation of $\mathrm{B}$ cells resulting in the stimulation of humoral immunity. In addition, it is important in the generation of Th2 effector cells from naïve CD4 T cells [26]. Moreover, IL-10, a regulatory cytokine involved in the stimulation of the humoral response, was also increased by $\mathrm{PppA}+\mathrm{LcV}, \mathrm{PppA}+\mathrm{LcM}, \mathrm{LcV}$ and LcM. Monocytes, macrophages, regulatory $\mathrm{T}$ and $\mathrm{T}$ helper (Th) cells are the major sources of IL-10 but other cell types, including natural killer (NK) cells, dendritic cells, cytotoxic T cells and B cells, also produce this cytokine [27,28]. IL-10 is typically an anti-inflammatory cytokine with regulatory functions of immune cells but it also plays a positive role in the promotion of humoral immunity since it enhances proliferation and differentiation of B cells, prevents their apoptosis and stimulates MHCII expression in these cells [29]. In addition, IL-10 stimulates NK activity and increases the IL-2-induced proliferation of CD56+ cells [30]. The stimulation of NK by live and heat-killed lactobacilli in "in vitro" studies was reported. Thus, live Lactobacillus casei Shirota (LcS) was able to enhance NK activity in PBMCs [31]. Besides, heat-killed LcS stimulated IL-10, IL-12 and TNF- $\alpha$ and promoted NK cells activity [32]. In our model, combinations of $\mathrm{LcV}+\mathrm{PppA}, \mathrm{LcM}+\mathrm{PppA}, \mathrm{LcV}$ and $\mathrm{LcM}$ increased IL-2 induction; thus, this fact, together with IL-10 increase, would be involved in NK stimulation. Numerous studies have demonstrated that cell components of lactobacilli such as LTA (lipoteichoic acid), lipopeptides, cell wall peptidoglycans $[33,34]$ through TLR2 in conjunction with TLR6 or TLR1 [35] and DNA [36,37] through TLR9 are able to stimulate the immune system. A recent report [38] has shown that NKT cells are required for protective responses against Streptococcus pneumoniae and other Gram positive pathogens. LcM increased this population in PBMCs and this fact would be benefic al for the development of a mucosal vaccine against pneumococci. In addition, LcV increased NKT population, although not signifi antly, with respect to the control group. However, both $\mathrm{LcV}+\mathrm{PppA}$ and LcM+PppA combinations were able to increase NKT population. The differences observed between $\mathrm{LcV}$ and $\mathrm{LcM}$ with respect to NKT (CD56+-CD3+) and NK cells (CD56+) would be due to alterations on the surface of Lactobacillus casei after it was heat killed; however, further studies are necessary to elucidate this point and to determine the mechanisms involved in this effect. On the other hand, both heat-killed and live lactobacilli as well as their respective combinations with the pneumococcal antigen stimulated IL-2 induction in PBMCs. Th s Th1 cytokine is very important in vaccination because it would influence the proliferation and maintenance of memory cells $[39,40]$, a desirable condition in vaccines with long-term efficacy. With respect to INF- $\gamma$, its patterns showed increased levels when PBMCs were stimulated with LcM and LcV, LcM reaching higher values than LcV. Th1 and NK cells are a source of INF- $\gamma$, which plays an important role in the neutrophilmediated host protective responses against pneumococcal infection 
promoted by NKT cells [41-43]. In addition, the combinations of PppA with LcV and LcM induced higher values of IL-17 cytokines. IL-17Asecreting CD4+ T cells (Th17) mediate resistance against mucosal colonization by $S$. pneumoniae and play an important role in the clearance of pathogens in a mouse model [44,45]. In addition, humans lacking Th17 cells due to genetic mutation are highly susceptible to mucosal infections by $S$. pneumoniae [46]. Scientific evidence in animal and human cell models indicates that Th17 cells are implicated in the defense against human pathogens. Recent reports have demonstrated that pneumococcal antigens able to activate Th17 cells are effective as mucosal immunogens that can afford protection to mice against $S$. pneumoniae colonization $[45,47]$. Th s protection occurs in a CD4+T cell and IL-17 dependent manner [45,47]. IL-17 induction is a desirable characteristic in the design of pneumococcal vaccines and both $\mathrm{LcV}+\mathrm{PppA}$ and $\mathrm{LcM}+\mathrm{PppA}$ combinations were able to induce this cytokine. However, the use of dead lactobacilli as adjuvants in mucosal vaccines is undoubtedly an advantage over other adjuvants because it would allow the safe administration of potential vaccines even in the case of immuno compromised hosts and elderly people without the risks associated with live bacteria [48]. On the other hand, some works have shown that "in vitro" assays do not necessarily correlate with clinical outcome $[49,50]$. In contrast, the results obtained in this paper with respect to the activation of Th1 and Th2 and also Th17 cells did correlate with the results obtained "in vivo" using a pneumococcal infection model and PppA+LcM and PppA+LcV combinations as a potential nasal vaccine [15]. To the best of our knowledge, this is the fi st work that evaluates the effect of a live or heat-killed lactic acid bacteria associated with a pneumococcal antigen in a human cell model and correlates the results with the nasal experimental vaccine evaluated in a mouse model. Adjuvant capacity is strain-dependent and specific antigens and their characteristics can induce different responses. Further studies are under way to evaluate the adjuvanticity of other lactic acid bacteria associated with viral and bacterial antigens. On the other hand, the immune response induced after vaccination depends on a multiplicity of factors such as nutritional status, preexisting immunity, genetic and environmental factors and geographical location of the host. Therefore, "in vitro" assays using human PBMC cultures that implicitly include the above aspects would be a useful model to evaluate an experimental mucosal vaccine that includes a lactic acid strains as adjuvant associated with specific antigens. The immune response of PBMCs after stimulation is more likely to mimic an "in vivo" response in human individuals than cell lines or isolated mucosal cells.

\section{Acknowledgements}

This work was supported by grants from CONICET PIP 632, CIUNT: D/403-3 and Préstamo BID-PICT2010 No 828

\section{References}

1. Dockrell DH, Whyte MK, Mitchell TJ (2012) Pneumococcal pneumonia: mechanisms of infection and resolution. Chest 142: 482-491.

2. Varon E (2012) Epidemiology of Streptococcus pneumoniae. Med Mal Infect 42: $361-365$

3. Godfroid F, Hermand P, Verlant V, Denoël P, Poolman JT (2011) Preclinical evaluation of the Pht proteins as potential cross-protective pneumococcal vaccine antigens. Infect Immun 79: 238-245

4. Rolo D, Ardanuy C, Fleites A, Martín R, Liñares J (2009) Diversity of pneumococcal surface protein A (PspA) among prevalent clones in Spain. BMC Microbiol 9: 80.

5. Holmgren J, Czerkinsky C (2005) Mucosal immunity and vaccines. Nat Med 11: S45-S53

6. Lawson LB, Norton EB, Clements JD (2011) Defending the mucosa: adjuvant and carrier formulations for mucosal immunity. Curr Opin Immunol 23: 414-420.
7. Norton EB, Lawson LB, Freytag LC, Clements JD (2011) Characterization of a mutant Escherichia coli heat-labile toxin, LT (R192G/L211A), as a safe and effective oral adjuvant. Clin Vaccine Immunol 18: 546-551.

8. Perdigon G, Alvarez S, Medina M, Vintiñi E, Roux E (1999) Influence of the oral administration of lactic acid bacteria on IgA producing cells associated to bronchus. Int J Immunopathol Pharmacol 12: 97-102.

9. de Moreno de LeBlanc A, Maldonado Galdeano C, Chaves S, Perdigon G (2005) Oral administration of L. casei CRL 431 increases immunity in bronchus and mammary glands. Eur J Inflamm 3: 25-30.

10. Clancy R (2003) Immunobiotics and the probiotic evolution. FEMS Immuno Med Microbiol 38: 9-12.

11. Racedo S, Villena J, Medina M, Agüero G, Rodríguez V, et al. (2006) Lactobacillus casei administration reduces lung injuries in a Streptococcus pneumoniae infection. Microbes Infect 8: 2359-2366.

12. Villena J, Barbieri N, Salva S, Herrera M, Alvarez S (2009) Enhanced immune response to pneumococcal infection in malnourished mice nasally treated with heat-killed Lactobacillus casei. Microbiol Immunol 53: 636-646.

13. Hernani Mde L, Ferreira PC, Ferreira DM, Miyaji EN, Ho PL, et al. (2011) Nasa immunization of mice with Lactobacillus casei expressing the pneumococcal surface protein $C$ primes the immune system and decreases pneumococcal nasopharyngeal colonization in mice. FEMS Immunol Med Microbiol 62: 263272.

14. Wells JM, Mercenier A (2008) Mucosal delivery of therapeutic and prophylactic molecules using lactic acid bacteria. Nat Rev Microbiol 6: 349-362.

15. Vintiñi EO, Medina MS (2011) Host immunity in the protective response to nasa immunization with a pneumococcal antigen associated to live and heat-killed Lactobacillus casei. BMC Immunol 12: 46.

16. Rizzardini G, Eskesen D, Calder P, Capetti A, Jespersen L, et al. (2012) Evaluation of the immune benefits of two probiotic strains Bifidobacterium animalis ssp. lactis, BB- $12^{\circledR}$ and Lactobacillus paracasei ssp. paracasei, L. casei $431^{\circledR}$ in an influenza vaccination model: a randomised, double-blind, placebo-controlled study. Br J Nutr 107: 876-884

17. Medina M, Villena J, Vintiñi E, Hebert EM, Raya R, et al. (2008) Nasa immunization with Lactococcus lactis expressing the pneumococcal protective protein A induces protective immunity in mice. Infect Immun 76: 2696-2705.

18. Vintiñi E, Villena J, Alvarez S, Medina M (2010) Administration of a probiotic associated with nasal vaccination with inactivated Lactococcus lactis-PppA induces effective protection against pneumoccocal infection in young mice. Clin Exp Immunol 159: 351-362.

19. Medina M, De Palma G, Ribes-Koninckx C, Calabuig M, Sanz Y (2008) Bifidobacterium strains suppress in vitro the pro-inflammatory milieu triggered by the large intestinal microbiota of coeliac patients. J Inflamm (Lond) 5: 19.

20. Medina M, Vintiñi E, Villena J, Raya R, Alvarez S (2010) Lactococcus lactis as an adjuvant and delivery vehicle of antigens against pneumococcal respiratory infections. Bioeng Bugs 1: 313-325

21. Woodrow KA, Bennett KM, Lo DD (2012) Mucosal Vaccine Design and Delivery. Annu Rev Biomed Eng 14: 17-46.

22. Fujkuyama Y, Tokuhara D, Kataoka K, Gilbert RS, McGhee JR, et al. (2012) Novel vaccine development strategies for inducing mucosal immunity. Expert Rev Vaccines 11: 367-379.

23. Maino VC, Suni MA, Ruitenberg JJ (1995) Rapid flow cytometric method for measuring lymphocyte subset activation. Cytometry 20: 127-133.

24. Caruso A, Licenziati S, Corulli M, Canaris AD, De Francesco MA, et al. (1997) Flow cytometric analysis of activation markers on stimulated $T$ cells and their correlation with cell proliferation. Cytometry 27: 71-76.

25. Antas PR, Oliveira EB, Milagres AS, Franken KC, Ottenhoff TH, et al. (2002) Kinetics of $\mathrm{T}$ cell activation molecules in response to Mycobacterium tuberculosis antigens. Mem Inst Oswaldo Cruz 97: 1097-1099.

26. Nelms K, Keegan AD, Zamorano J, Ryan JJ, Paul WE (1999) The IL-4 receptor: signaling mechanisms and biologic functions. Annu Rev Immunol 17: 701-738.

27. Saraiva M, O'Garra A (2010) The regulation of IL-10 production by immune cells. Nat Rev Immunol 10: 170-181.

28. Sabat R, Grütz G, Warszawska K, Kirsch S, Witte E, et al. (2010) Biology of interleukin-10. Cytokine Growth Factor Rev 21: 331-344. 
Citation: Vintiñi E, Gonzalez L, Medina M (2012) Specific Immune Response Induced by a Lactobacillus Associated with a Pneumococcal Antigen in an "in vitro" Human Cells Model. J Vaccines Vaccin 3:153. doi:10.4172/2157-7560.1000153

29. Burdin N, Rousset F, Banchereau, J (1997) B-cell-derived IL-10: production and function. Methods 11: 98-111.

30. Carson WE, Lindemann MJ, Baiocchi R, Linett M, Tan JC, et al. (1995) The functional characterization of interleukin-10 receptor expression on human natural killer cells. Blood 85: 3577-3585.

31. Takeda K, Suzuki T, Shimada SI, Shida K, Nanno M, et al. (2006) Interleukin-12 is involved in the enhancement of human natural killer cell activity by Lactobacillus casei Shirota. Clin Exp Immunol 146: 109-115.

32. Shida K, Suzuki T, Kiyoshima-Shibata J, Shimada S, Nanno M (2006) Essential roles of monocytes in stimulating human peripheral blood mononuclear cells with Lactobacillus casei to produce cytokines and augment natural killer cell activity. Clin Vaccine Immunol 13: 997-1003.

33. Kang SS, Ryu YH, Baik JE, Yun CH, Lee K, et al. (2011) Lipoteichoic acid from Lactobacillus plantarum induces nitric oxide production in presence of interferon-in murine macrophages. Mol Immunol 48: 2170-2177.

34. Chen T, Isomäki P, Rimpiläinen M, Toivanen P (1999) Human cytokine responses induced by gram-positive cell walls of normal intestinal microbiota. Clin Exp Immunol 118: 261-267.

35. Heine $H$, Lien E (2003) Toll-like receptors and their function in innate and adaptive immunity. Int Arch Allergy Immunol 130: 180-192.

36. Lammers KM, Brigidi P, Vitali B, Gionchetti P, Rizzello F, et al. (2003) Immunomodulatory effects of probiotic bacteria DNA: IL-1 and IL-10 response in human peripheral blood mononuclear cells. FEMS Immunol Med Microbiol 38: 165-172.

37. Rachmilewitz D, Katakura K, Karmeli F, Hayashi T, Reinus C, et al. (2004) Tolllike receptor 9 signaling mediates the anti-inflammatory effects of probiotics in murine experimental colitis. Gastroenterology 126: 520-528.

38. Girardi E, Yu ED, Li Y, Tarumoto N, Pei B, et al. (2011) Unique interplay between sugar and lipid in determining the antigenic potency of bacterial antigens for NKT cells. PLoS Biol 9: e1001189.

39. Bejon P, Keating S, Mwacharo S, Kai OK, Dunachie S, et al. (2006) Early gamma interferon and interleukin-2 responses to vaccination predict the late resting memory in malaria-naïve and malaria-exposed individuals. Infect Immun 74: 6331-6338.
40. Foulds KE, Wu CY, Seder RA (2006) Th1 memory: implications for vaccine development. Immunol Rev 211: 58-66.

41. Nakamatsu M, Yamamoto N, Hatta M, Nakasone C, Kinjo T, et al. (2007) Role of interferon-gamma in Valpha14+ natural killer T cell-mediated host defense against Streptococcus pneumoniae infection in murine lungs. Microbes Infect 9: 364-374.

42. Kinjo Y, Illarionov P, Vela JL, Pei B, Girardi E, et al. (2011) Invariant natura killer T cells recognize glycolipids from pathogenic Gram-positive bacteria. Nat Immunol 12: 966-974.

43. Subrahmanyam P, Sun W, East J, Li J, Webb TJ (2012) Natural Killer T Cell Based Immunotherapy. J Vaccines Vaccin 3: 4

44. Moffitt KL, Gierahn TM, Lu YJ, Gouveia P, Alderson M, et al. (2011) T (H)17based vaccine design for prevention of Streptococcus pneumoniae colonization. Cell Host Microbe 9: 158-165.

45. Marqués JM, Rial A, Muñoz N, Pellay FX, Van Maele L, et al. (2012) Protection against Streptococcus pneumoniae serotype 1 acute infection shows a signature of Th17- and IFN-y-mediated immunity. Immunobiol 217: 420-429.

46. Milner JD, Brenchley JM, Laurence A, Freeman AF, Hill BJ, et al. (2008) Impaired $T(H) 17$ cell differentiation in subjects with autosomal dominant hyperIgE syndrome. Nature 452: 773-776.

47. Zhang Z, Clarke TB, Weiser JN (2009) Cellular effectors mediating Th17dependent clearance of pneumococcal colonization in mice. J Clin Invest 119 1899-1909.

48. Detmer A, Glenting J (2006) Live bacterial vaccines-a review and identification of potential hazards. Microb Cell Fact 5: 23.

49. Flinterman AE, Knol EF, van leperen-van Dijk AG, Timmerman HM, Knulst AC et al. (2007) Probiotics have a different immunomodulatory potential in vitro versus ex vivo upon oral administration in children with food allergy. Int Arch Allergy Immunol 143: 237-244.

50. Kopp MV, Goldstein M, Dietschek A, Sofke J, Heinzmann A, et al. (2008) Lactobacillus GG has in vitro effects on enhanced interleukin-10 and interferongamma release of mononuclear cells but no in vivo effects in supplemented mothers and their neonates. Clin Exp Allergy 38: 602-610. 